\title{
Awareness, knowledge, attitudes and practices on the management of diabetes mellitus patients with periodontitis amongst Malaysian primary care practitioners
}

\author{
Norul Nurdiyana Nordin, Rathna Devi Vaithilingam, Roslan Saub, Nazrila Hairizan Nasir, \\ Ahmad Sharifuddin Mohd Asari, Baizury Bashah, Maimunah Mahmud, Nik Mazlina \\ Mohammad \\ Nordin NN, Vaithilingam RD, Saub R, et al. Awareness, knowledge, attitudes and practices on the management of diabetes mellitus patients \\ with periodontitis amongst Malaysian primary care practitioners. Malays Fam Physician. 2021;16(3);44-55. https://doi.org/10.51866/oa1131
}

\section{Keywords:}

Diabetes, periodontitis,

knowledge, awareness,

medical practitioners

\section{Authors:}

Rathna Devi Vaithilingam

(Corresponding author)

BDS (Malaya), MClinDent (Malaya)

Deparment of Restorative Dentistry

Faculty of Dentistry, University of

Malaya, Kuala Lumpur, Malaysia

Email: rathna@um.edu.my

Norul Nurdiyana Nordin

DDS (UKM)

Department of Restorative Dentistry

Faculty of Dentistry, University of

Malaya, Kuala Lumpur, Malaysia

\section{Roslan Saub}

BDS (Malaya), MDSc (Melb), PhD

(Toronto)

Deparment of Community Oral

Health and Clinical Prevention

Faculty of Dentistry, University of

Malaya, Kuala Lumpur, Malaysia

\section{Nazrila Hairizan Nasir}

MD (UKM), M Med (Fam Med)

(Malaya), Msc Child Mental Health

(University of Leceister, UK)

Bahagian Pembangunan Kesihatan

Keluarga, Cawangan Kesihatan

Primer, Putrajaya, Malaysia

\begin{abstract}
Introduction: The bi-directional relationship between periodontitis (PD) and diabetes mellitus (DM) has been confirmed. Medical practitioners (MPs) serve an important role in relaying this information to patients. This study aimed to investigate the awareness, knowledge, attitudes and practices (KAP) in the management of DM patients with $\mathrm{PD}$ in primary care clinics under the Ministry of Health (MOH) of Malaysia.

Materials and Methods: A self-administered questionnaire on KAP in the management of DM patients with PD was posted to 725 medical officers (MOs) and family medicine specialists (FMSs) in $\mathrm{MOH}$ clinics in Kedah, Terengganu, Johor and Negeri Sembilan. Collected data were tabulated and analysed using descriptive and regression analyses (simple and multiple). Statistical significance was defined as $\mathrm{p}<0.05$.

Results: A total of 549 MPs responded. The majority of MPs were MOs (92.6\%) and female $(75.8 \%)$. FMSs had a greater awareness of PD when compared to MOs $(\mathrm{p}=0.002)$. All MPs had good knowledge, except for the incorrect notion that excessive sugar causes PD (94.3\%). Overall, FMSs had better knowledge when compared to MOs $(\mathrm{p}=0.026)$. The majority of MPs agreed that 'they should update their knowledge on the association between systemic disease and PD' (89.6\%) and claimed that 'it was not their responsibility to examine DM patients for PD' (83.1\%). Most MPs did not enquire or examine for PD in their DM patients. More FMSs (67.5\%) referred patients to dentists compared to MOs (31.6\%).

Conclusion: Most MPs have sufficient knowledge on PD, but a negative attitude in the management of PD in DM patients. The reasons for not referring included workload and patients refusing referral.
\end{abstract}

\section{Introduction}

Periodontitis (PD) is a chronic inflammatory disease affecting tooth-supporting structures. It causes the loss of periodontal attachment and progressive alveolar bone loss, which results in tooth loss. Severe PD is the sixth most prevalent disease worldwide, with a prevalence of $11.2 \%$ (approximately 743 million people affected).

The bi-directional relationship between PD and DM has been widely accepted. Poorly controlled DM patients may have a higher risk of developing severe PD when compared to non-DM controls. ${ }^{2}$ Conversely, PD patients with DM exhibit poorer glycaemic control and a higher presence of diabetesrelated complications. ${ }^{3}$ Following periodontal therapy, reductions in glycated haemoglobin (HbA1c; ranging from 0.27 to $0.48 \%$ ) have been reported at 3 months post-therapy, ${ }^{4}$ which are equivalent to those achieved by adding a second medication to the diabetes pharmacological regime. ${ }^{5}$

Most DM patients in Malaysia are managed by medical practitioners (MPs) in governmentrun primary health clinics based on the Malaysian Clinical Practice Guidelines (CPGs) for the management of DM patients. ${ }^{6}$ MPs must have knowledge and awareness of the dynamics of these two important diseases 


\section{Ahmad Sharifuddin Mohd Asari BDS (Egypt), M.Sc (Peridontology) (London), FDSRCPS (Glasgow) Faculty of Dentistry, UiTM Kampus Sungai Buloh, Sungai Buloh, Selangor Malaysia}

\section{Baizury Bashah}

MBBS (Malaya), M Med (Fam Med) (Malaya)

Klinik Kesihatan Kuala Lumpur

Wilayah Persekutuan Kuala Lumpur Malaysia

\section{Maimunah Mahmud}

MBBS (Malaya), M Med (Fam Med) (Malaya)

Klinik Kesihatan Sungai Buloh Sungai Buloh, Selangor, Malaysia

\section{Nik Mazlina Mohammad}

MBBS (Malaya), M Med (Fam Med) (Malaya)

Klinik Kesihatan Kelana Jaya Petaling Jaya, Selangor, Malaysia to help in the overall management of their patients. However, this information is lacking in the current edition (2015) of the CPG.

Studies have reported that awareness could be associated with better practices. ${ }^{7,8}$ Attitudes are also expected to serve an important role in the outcomes of clinical care in the management of DM patients with PD. ${ }^{9,10}$ A qualitative study in the United Kingdom concluded that the knowledge of DM nurses, dental professionals and medical doctors were limited regarding the link between both diseases. ${ }^{11}$ Similar findings were also reported in India and Jordan. ${ }^{8,12,13}$ In contrast, family medicine specialists (FMSs) in Hong Kong reported that the majority (92\%) of them were aware of the effects of poor glycaemic control on PD; however, only $76 \%$ were aware of the reverse effect that PD has on DM $(p=0.02)$. In terms of their clinical practice, only $5.7 \%$ routinely asked patients about their dental history, whilst $7.1 \%$ often examined their patients' mouths and 12.1\% recommended that their patients visit a dentist. It was shown that the more knowledgeable the MPs were, the more likely they were to make dental referrals. ${ }^{7}$

It is important to understand the barriers to identify opportunities that may help to improve the quality of diabetes care, such as the improvement of metabolic control and diabetes self-management.14 It has previously been postulated that it is not the MP's responsibility to perform oral examinations. ${ }^{?}$ Moreover, MPs may have received limited training in oral health knowledge/education in medical school. ${ }^{10}$

In 2017, Salleh et al. assessed the perceptions and practices of Malaysian medical personnel—including medical officers (MOs) and FMSs - in referring DM patients to receive dental care. ${ }^{15}$ However, they did not assess the knowledge and awareness of these MPs in the DM management of patients with PD. Therefore, the present study aimed to assess the awareness, knowledge, attitudes and practices (KAP) of Malaysian MPs in the DM management of patients with PD and identify the associated barriers.

\section{Methods}

This was a cross-sectional study conducted on MPs in the Ministry of Health (MOH) of Malaysia. Ethical clearance for this study was granted by the Medical Ethics Committee, Faculty of Dentistry, University of Malaya (reference number: DF RD1718/0059(P)) and the Medical Research and Ethics Committee, Ministry of Health, Malaysia (NMRR-172830-38175).

A self-administered questionnaire survey was distributed to MOs and FMSs practising in the four states (Kedah, Terengganu, Johor and Negeri Sembilan) with the highest prevalence of DM in Malaysia based on the National Health and Morbidity Survey 2015. ${ }^{16}$ The convenience sampling method was used in this study and the study population comprised all MPs who were involved in diabetic patient management in all primary care clinics with resident FMSs in the four respective states. MPs who practised in clinics without resident FMSs were excluded. Sample size estimation was performed based on the previous literature ${ }^{8,17}$. It was found that $70 \%$ of the medical doctors reportedly knew about the relationship between diabetes and periodontal disease. Thus, the expected prevalence for the calculation was $70 \%$. The acceptable sample size was 386 , including a $20 \%$ dropout rate.

The questions in the questionnaire were adapted from several studies for each domain and measured ${ }^{7,8,15,18}$ as follows:

1) Demographics - Seven questions related to the respondent's background.

2) Social and oral health habits - Four questions on the respondent's social and oral health habits as well as their perception of their oral health. Responses are scored on a five-point scale (very good, good, fair, poor and very poor).

3) Awareness of the PD-DM bi-directional relationship - Three questions covering the association of uncontrolled diabetes with $\mathrm{PD}$ and interventions/treatments that may control diabetic status. For this domain, there were three possible responses for each item: 'yes', 'no' and 'do not know'. Each correct answer to a question was given a score of 1, whilst an incorrect or 'do not know' answer was given a score of 0 . The overall score was the sum of the scores for the three questions and ranged from 0 to 3, with higher scores indicating better awareness. For the assessment of awareness level, the respondents were divided into good and poor awareness groups based on their mean awareness scores. 
4) Knowledge of PD - Eight questions were related to knowledge of $\mathrm{PD}$, knowledge of causative factors/modifying factors of PD and knowledge of the signs and symptoms of PD. The response options for each item were 'yes', 'no' and 'do not know'. Each correct answer to a question was given a score of 1 , whilst an incorrect or 'do not know' answer was given a score of 0 . The overall score was the sum of the scores for the eight questions and ranged from 0 to 8 , with higher scores indicating better knowledge. The respondent's level of knowledge was also categorised into good or poor based on their mean knowledge score (Table 2).

5) Attitudes towards the management of DM patients with PD - Five questions were related to the respondent's attitudes towards the management of diabetic patients with PD and covered attitudes towards updating knowledge on diabetes mellitus and PD, identifying PD, educating diabetic patients about PD and referring diabetic patients to dental clinics. The attitude questions were scored on a 5-point Likert scale: 1 point for 'strongly disagree', 2 for 'disagree', 3 for 'neither agree nor disagree', 4 for 'agree' and 5 for 'strongly agree'. Depending on the statements, each response was then categorised into having a positive attitude (score of 1 ) or a negative attitude (score of 0 ). The overall attitude score was the sum of the scores for the five questions and ranged from 0 to 5, with higher scores indicating a better attitude. The respondents' attitudes towards their management of diabetic patients with periodontal disease were categorised into positive or negative based on their mean attitude scores.

6) Practice behaviour in the management of DM patients with PD - Three questions related to practices covered enquiries about patients' oral health, the examination of patients' oral health and the referral of diabetic patients to a dental clinic. For this domain, there were three possible responses for each item: 'yes', 'no' and 'do not know'. Positive practices were given a score of 1 , whilst negative practices or 'no' answers were given a score of 0 . The overall score was the sum of the scores for the three questions and ranged from 0 to 3 , with higher scores indicating better practices. The respondents' practices in their management of diabetic patients with periodontal disease were divided into good and poor practices based on their mean practice scores (Table 2).

7) Barriers to the management of DM patients with periodontal disease - Six questions were related to the barriers experienced by MPs in their management of diabetic patients with periodontal disease. These barriers included patient factors, healthcare professional factors and health service factors. The respondents were asked to identify barriers in their management of DM with periodontal disease.

Content validation was performed with one dental public health specialist and three periodontists. Pre-testing of the questionnaire was then performed with 10 FMSs and MOs at Klinik Kesihatan Sungai Buloh for face validation. These FMSs and MOs were not involved with the final survey. A total of 725 questionnaires were distributed from September to December 2018. The distribution and collection of survey forms were performed through state representatives.

\section{Data Analysis}

Data were described using the means and standard deviations for continuous variables and the frequency distributions for categorical variables. In this study, the dependent variables were the level of awareness, knowledge, attitudes and practices. Simple and multiple logistic regression analyses were performed to investigate potential relationships between demographic variables (i.e., age, gender, ethnicity, profession and years of service), smoking status and perceived oral health status with the level of awareness, knowledge, attitude and practice scores. In the logistic regression analysis, a dummy variable was created for categorical independent variables with more than two categories and identified reference options. Adjusted p-values were considered significant at the 0.05 level. All analyses were performed using Statistical Package for Social Science (SPSS), version 20.

\section{Results}

Out of the 725 questionnaires distributed, 549 (75\%) were returned. Three questionnaires were excluded due to incomplete data. A total of $506 \mathrm{MOs}(92.7 \%)$ and 40 FMSs $(7.3 \%)$ 
responded. The mean age of MPs was $33.31(\mathrm{SD}=5.9)$ years, with a range of $25-56$ years. The majority of MPs were female (75.8\%) and Malay (80.6\%).

Table 1 presents the distribution of MPs' responses regarding their awareness of the relationship between DM and PD. Most MPs were aware of the bi-directional relationship between DM and PD and that uncontrolled DM patients had a higher risk of developing PD. When compared to MOs, FMSs had better awareness of the effect of periodontal treatment on DM patients' glycaemic control.

Table 1. Percentage distribution of the awareness status of MPs regarding the relationship between $\mathrm{DM}$ and PD.

\begin{tabular}{|c|c|c|c|}
\hline Awareness statement & $\begin{array}{l}\text { Medical officers } \\
\begin{array}{c}(\mathrm{N}=506) \\
\mathrm{n}(\%)\end{array}\end{array}$ & $\begin{array}{c}\text { Family medicine } \\
\text { specialists }(\mathrm{N}=40) \\
\mathrm{n}(\%)\end{array}$ & $\begin{array}{c}\text { Total }(\mathrm{N}=546) \\
\mathrm{n}(\%)\end{array}$ \\
\hline $\begin{array}{l}\text { Q1: There is a bi-directional } \\
\text { relationship between DM and } \\
\text { periodontal disease. } \\
\text { Yes } \\
\text { No }\end{array}$ & $\begin{array}{l}403(79.6) \\
103(20.4)\end{array}$ & $\begin{array}{c}37(92.5) \\
3(7.5)\end{array}$ & $\begin{array}{l}440(80.6) \\
106(19.4)\end{array}$ \\
\hline $\begin{array}{l}\text { Q2: Uncontrolled diabetic } \\
\text { patients have a higher risk } \\
\text { of developing periodontal } \\
\text { disease. } \\
\text { Yes } \\
\text { No }\end{array}$ & $\begin{array}{c}475(93.9) \\
31(1.8)\end{array}$ & $\begin{array}{c}40(100) \\
0\end{array}$ & $\begin{array}{c}515(94.7) \\
31(5.6)\end{array}$ \\
\hline $\begin{array}{l}\text { Q3: Periodontal treatment can } \\
\text { improve the glycaemic control } \\
\text { of a diabetic patient. } \\
\text { Yes } \\
\text { No }\end{array}$ & $\begin{array}{l}203(40.1) \\
303(30.8)\end{array}$ & $\begin{array}{l}25(62.5) \\
15(37.5)\end{array}$ & $\begin{array}{l}228(41.8) \\
318(58.2)\end{array}$ \\
\hline
\end{tabular}

The distributions and mean KAP scores of the MPs are presented in Table 2.

Table 2. Status and mean scores of MPs' awareness, knowledge, attitudes and practices related to the management of DM patients with PD.

\begin{tabular}{|c|c|c|c|}
\hline Domain & $\begin{array}{l}\text { Medical officers } \\
\quad(\mathrm{N}=506)\end{array}$ & $\begin{array}{c}\text { Family medicine } \\
\text { specialists }(\mathrm{N}=40)\end{array}$ & $p$-value \\
\hline $\begin{array}{l}\text { Awareness } \\
\text { Status } \\
\text { Good }[\mathrm{n}(\%)] \\
\text { Poor }[\mathrm{n}(\%)] \\
\text { Total score }[\text { Mean }(\mathrm{SD})]\end{array}$ & $\begin{array}{c}413(81.6) \\
93(18.4) \\
2.14(0.82)\end{array}$ & $\begin{array}{c}39(98.9) \\
1(2.5) \\
2.55(0.5)\end{array}$ & $\begin{array}{l}{ }^{*} 0.01 \\
{ }^{*} 0.02\end{array}$ \\
\hline $\begin{array}{l}\text { Knowledge } \\
\text { Status } \\
\text { Good }[\mathrm{n}(\%)] \\
\text { Poor }[\mathrm{n}(\%)] \\
\text { Total score }[\text { Mean }(\mathrm{SD})]\end{array}$ & $\begin{array}{c}492(97.2) \\
14(2.8) \\
2.14(0.82)\end{array}$ & $\begin{array}{c}40(100) \\
0(0) \\
2.55(0.5)\end{array}$ & $\begin{array}{l}0.29 \\
0.28 \\
\end{array}$ \\
\hline $\begin{array}{l}\text { Attitude } \\
\text { Status } \\
\text { Positive }[\mathrm{n}(\%)] \\
\text { Negative }[\mathrm{n}(\%)] \\
\text { Total score }[\text { Mean }(\mathrm{SD})]\end{array}$ & $\begin{array}{c}157(31) \\
349(69) \\
2.26(0.86)\end{array}$ & $\begin{array}{c}15(37.5) \\
25(62.5) \\
2.43(0.84)\end{array}$ & $\begin{array}{c}0.39 \\
0.8 \\
\end{array}$ \\
\hline $\begin{array}{l}\text { Practice } \\
\text { Status } \\
\text { Positive }[\mathrm{n}(\%)] \\
\text { Negative }[\mathrm{n}(\%)] \\
\text { Total score }[\text { Mean(SD)] }\end{array}$ & $\begin{array}{l}121(23.9) \\
385(76.1) \\
0.81(1.06)\end{array}$ & $\begin{array}{c}19(47.5) \\
21(52.5) \\
1.45(1.20)\end{array}$ & $\begin{array}{l}{ }^{*} 0.01 \\
{ }^{*} 0.00\end{array}$ \\
\hline
\end{tabular}

a: Pearson's chi-squared test, *: Significant difference observed between groups at $\mathrm{p}<0.05$. 
Table 3 presents the distribution of MPs' responses regarding their knowledge of PD. Most of the MPs had good knowledge of PD, except for knowledge on whether excessive sugar intake could cause PD since most of the MPs either did not know or chose the incorrect answer. Of the $546 \mathrm{MPs}$, only $3.1 \%$ provided the correct response to this question (3\% of the MOs and $5 \%$ of the FMSs). For the assessment of MPs' knowledge, MPs were divided into good and poor knowledge groups (Table 2). Overall, $97.2 \%$ of MOs and 100\% of FMSs exhibited good knowledge, with mean scores of $6.63(\mathrm{SD}=0.78)$ and $6.8(\mathrm{SD}=0.52)$ respectively. However, there was no significant difference between the good and poor knowledge score groups for MOs and FMSs $(p=0.29)$. There was also no significant difference in the total knowledge score between FMSs and MOs $(p=0.28)$.

Table 3. Percentage distribution of MPs' knowledge on PD.

\begin{tabular}{|c|c|c|c|}
\hline Knowledge statement & $\begin{array}{l}\text { Medical officers } \\
\begin{array}{c}(\mathrm{N}=506) \\
\mathrm{n}(\%)\end{array}\end{array}$ & $\begin{array}{l}\text { Family medicine } \\
\text { specialists }(\mathbf{N}=40) \\
\mathbf{n}(\%)\end{array}$ & $\begin{array}{c}\text { Total }(\mathrm{N}=546) \\
\mathrm{n}(\%)\end{array}$ \\
\hline $\begin{array}{l}\text { Q1: Dental plaque can cause } \\
\text { periodontal disease. } \\
\text { Yes } \\
\text { No }\end{array}$ & $\begin{array}{c}480(94.8) \\
26(5.2)\end{array}$ & $\begin{array}{c}38(95) \\
2(5)\end{array}$ & $\begin{array}{c}518(94.9) \\
28(5.1)\end{array}$ \\
\hline $\begin{array}{l}\text { Q2: Gingivitis appears clinically as } \\
\text { inflammation of the gingiva } \\
\text { (gums). } \\
\text { Yes } \\
\text { No }\end{array}$ & $\begin{array}{c}503(99.4) \\
3(0.6)\end{array}$ & $\begin{array}{c}40(100) \\
0\end{array}$ & $\begin{array}{c}543(99.5) \\
3(0.6)\end{array}$ \\
\hline $\begin{array}{l}\text { Q3: Smoking is a modifying risk } \\
\text { factor for periodontitis. } \\
\text { Yes } \\
\text { No }\end{array}$ & $\begin{array}{c}493(97.4) \\
13(2.6)\end{array}$ & $\begin{array}{c}40(100) \\
0\end{array}$ & $\begin{array}{c}533(97.6) \\
13(0.4)\end{array}$ \\
\hline $\begin{array}{l}\text { Q4: Excessive sugar intake can } \\
\text { cause periodontitis. } \\
\text { Yes } \\
\text { No }\end{array}$ & $\begin{array}{c}477(94.3) \\
29(5.7)\end{array}$ & $\begin{array}{c}38(95) \\
2(5)\end{array}$ & $\begin{array}{c}515(94.3) \\
31(5.7)\end{array}$ \\
\hline $\begin{array}{l}\text { Q5: Bleeding from the gingiva } \\
\text { (gums) can be a sign of } \\
\text { periodontitis. } \\
\text { Yes } \\
\text { No }\end{array}$ & $\begin{array}{c}462(91.3) \\
44(8.7)\end{array}$ & $\begin{array}{c}40(100) \\
0\end{array}$ & $\begin{array}{c}502(91.9) \\
44(8.1)\end{array}$ \\
\hline $\begin{array}{l}\text { Q6: Periodontitis can cause teeth } \\
\text { to become mobile. } \\
\text { Yes } \\
\text { No }\end{array}$ & $\begin{array}{c}438(86.6) \\
68(13.4)\end{array}$ & $\begin{array}{c}39(97.5) \\
1(2.5)\end{array}$ & $\begin{array}{c}477(87.4) \\
69(12.6)\end{array}$ \\
\hline $\begin{array}{l}\text { Q7: Gingival/gum swelling may be } \\
\text { a symptom of periodontitis. } \\
\text { Yes } \\
\text { No }\end{array}$ & $\begin{array}{c}490(96.8) \\
16(3.2)\end{array}$ & $\begin{array}{c}39(97.5) \\
1(2.5)\end{array}$ & $\begin{array}{c}529(96.9) \\
17(3.1)\end{array}$ \\
\hline $\begin{array}{l}\text { Q8: Halitosis (bad breath) may be } \\
\text { a symptom of periodontitis. } \\
\text { Yes } \\
\text { No }\end{array}$ & $\begin{array}{c}476(94.1) \\
30(5.9)\end{array}$ & $\begin{array}{c}37(92.5) \\
3(7.5)\end{array}$ & $\begin{array}{c}513(94) \\
33(6)\end{array}$ \\
\hline
\end{tabular}

Table 4 presents the distribution of MPs' responses regarding their attitudes in the management of DM patients with PD. The majority of the MPs agreed to the statements 'overall health cannot affect periodontal health' $(81.4 \%)$, 'it is not their responsibility to look into a patient's mouth to detect periodontal problems', 'they should update their knowledge on the association between systemic disease and PD' and 'they should help to educate DM patients on their increased risk of getting PD'. Regarding the need for all DM patients to receive a dental referral, half of the MPs agreed with the statement that there was no need for them to refer all DM patients to a dentist. 
Overall, $31 \%$ of MOs and $37.5 \%$ of FMSs exhibited positive attitudes, with mean scores of 2.26 $(\mathrm{SD}=0.85)$ and $2.43(\mathrm{SD}=0.84)$, respectively $(\mathrm{p}>0.05)($ Table 2$)$. No significant differences were observed in the attitude scores between FMSs and MOs.

Table 4. Distribution of MPs' attitudes in their management of diabetic patients.

\begin{tabular}{|c|c|c|c|}
\hline Attitude statement & $\begin{array}{l}\text { Medical officers } \\
\left(\begin{array}{c}\mathbf{N}=506) \\
\mathbf{n}(\%)\end{array}\right.\end{array}$ & $\begin{array}{l}\text { Family medicine } \\
\text { specialists } \\
(\mathbf{N}=40) \\
\mathbf{n}(\%)\end{array}$ & $\begin{array}{c}\text { Total }(\mathbf{N}=546) \\
\mathbf{n}(\%)\end{array}$ \\
\hline $\begin{array}{l}\text { Q1: I don't feel that overall health can } \\
\text { affect periodontal health. } \\
\text { Fully disagree } \\
\text { Somewhat disagree } \\
\text { I do not know } \\
\text { Somewhat agree } \\
\text { Fully agree }\end{array}$ & $\begin{array}{c}22(4.4) \\
57(11.3) \\
20(4) \\
175(34.7) \\
232(45.8)\end{array}$ & $\begin{array}{c}1(2.5) \\
2(5) \\
0(0) \\
10(25) \\
27(67.5)\end{array}$ & $\begin{array}{c}23(4.2) \\
59(10.8) \\
20(3.7) \\
185(34) \\
259(47.4)\end{array}$ \\
\hline $\begin{array}{l}\text { Q2: It is not my responsibility to look } \\
\text { into a patient's mouth to detect a } \\
\text { periodontal problem. } \\
\text { Fully disagree } \\
\text { Somewhat disagree } \\
\text { I do not know } \\
\text { Somewhat agree } \\
\text { Fully agree }\end{array}$ & $\begin{array}{c}12(2.4) \\
72(14.2) \\
52(10.3) \\
174(34.4) \\
196(38.7)\end{array}$ & $\begin{array}{c}1(2.5) \\
8(20) \\
1(2.5) \\
11(27.5) \\
19(47.5)\end{array}$ & $\begin{array}{c}13(2.4) \\
80(14.7) \\
53(9.7) \\
185(34) \\
215(39.4)\end{array}$ \\
\hline $\begin{array}{l}\text { Q3: I should update my knowledge on } \\
\text { the association between systemic } \\
\text { disease and periodontitis. } \\
\text { Fully disagree } \\
\text { Somewhat disagree } \\
\text { I do not know } \\
\text { Somewhat agree } \\
\text { Fully agree }\end{array}$ & $\begin{array}{c}24(4.7) \\
12(2.4) \\
17(3.4) \\
176(34.8) \\
277(54.7)\end{array}$ & $\begin{array}{c}1(2.5) \\
2(5) \\
1(2.5) \\
14(35) \\
22(55)\end{array}$ & $\begin{array}{c}25(4.9) \\
14(2.6) \\
18(3.3) \\
190(34.8) \\
299(54.8)\end{array}$ \\
\hline $\begin{array}{l}\text { Q4: I should help to educate diabetic } \\
\text { patients on their risk of getting } \\
\text { periodontitis. } \\
\text { Fully disagree } \\
\text { Somewhat disagree } \\
\text { I do not know } \\
\text { Somewhat agree } \\
\text { Fully agree }\end{array}$ & $\begin{array}{c}21(4.2) \\
15(3) \\
25(4.9) \\
177(35) \\
268(53)\end{array}$ & $\begin{array}{c}1(2.5) \\
1(2.5) \\
0(0) \\
13(32.5) \\
25(62.5)\end{array}$ & $\begin{array}{c}22(4) \\
16(2.9) \\
25(4.9) \\
190(34.8) \\
293(53.7)\end{array}$ \\
\hline $\begin{array}{l}\text { Q5: There is no need for dental referrals } \\
\text { for all diabetic patients. } \\
\text { Fully disagree } \\
\text { Somewhat disagree } \\
\text { I do not know } \\
\text { Somewhat agree } \\
\text { Fully agree }\end{array}$ & $\begin{array}{c}5(3) \\
67(13.2) \\
124(24.5) \\
148(29.2) \\
152(30)\end{array}$ & $\begin{array}{c}4(10) \\
7(9.5) \\
4(10) \\
9(22.5) \\
16(40)\end{array}$ & $\begin{array}{c}9(1.6) \\
74(13.6) \\
128(23.4) \\
157(27.1) \\
168(30.8)\end{array}$ \\
\hline
\end{tabular}

Regarding the MPs' practices in the management of DM patients, the majority of them did not ask their patients whether they had any symptoms of PD. In comparison, more FMSs examined and referred their patients for PD.

Overall, $76.1 \%$ of MOs with poor practices were represented by a mean practice score of $0.81(\mathrm{SD}=1.06)$ (Table 2$)$. There were significantly more 'poor practice' compared to 'good practice' scores in both groups ( $\mathrm{p}=$ 0.01 ). Moreover, mean total practice scores were significantly higher among the FMSs when compared to the MOs $(\mathrm{p}=0.00)$. 
Table 5. Percentage distribution of MPs' knowledge on PD.

\begin{tabular}{|c|c|c|c|}
\hline Practices questions & $\begin{array}{l}\text { Medical officers } \\
\left(\begin{array}{l}\mathrm{N}=506) \\
\mathbf{n}(\%)\end{array}\right.\end{array}$ & $\begin{array}{l}\text { Family medicine } \\
\text { specialists }(\mathrm{N}=40) \\
\mathrm{n}(\%)\end{array}$ & $\begin{array}{c}\text { Total }(\mathbf{N}=546) \\
\mathrm{n}(\%)\end{array}$ \\
\hline $\begin{array}{l}\text { Q1: Do you ask your patients } \\
\text { whether they have any } \\
\text { symptoms of periodontitis? } \\
\text { Yes } \\
\text { No }\end{array}$ & $\begin{array}{l}145(28.7) \\
361(71.3)\end{array}$ & $\begin{array}{l}20(50) \\
20(50)\end{array}$ & $\begin{array}{l}165(30.2) \\
381(69.8)\end{array}$ \\
\hline $\begin{array}{l}\text { Q2: Do you examine your patients } \\
\text { for signs of periodontitis? } \\
\text { Yes } \\
\text { No }\end{array}$ & $\begin{array}{l}104(20.6) \\
402(79.4)\end{array}$ & $\begin{array}{l}15(37.5) \\
25(62.5)\end{array}$ & $\begin{array}{l}119(21.8) \\
427(78.2)\end{array}$ \\
\hline $\begin{array}{l}\text { Q3: Do you refer your diabetic } \\
\text { patients to a dentist? } \\
\text { Yes } \\
\text { No }\end{array}$ & $\begin{array}{l}160(31.6) \\
346(68.4)\end{array}$ & $\begin{array}{l}27(67.5) \\
13(32.5)\end{array}$ & $\begin{array}{l}187(34.2) \\
359(65.8)\end{array}$ \\
\hline
\end{tabular}

Among all MPs, the greatest barrier to referral was workload, which was faced by more MOs than FMSs. The next barrier faced was patients refusing to be referred to a dentist (45.4\%), which was similarly noted by both MOs (45.1\%) and FMSs (50\%). Additional barriers were less frequently experienced by the MPs and included 'time consuming to write a referral letter to the dentist' (26.6\%), 'oral healthcare is not part of diabetes management in the CPG - Management of DM 2015 ' (15.8\%), 'dental clinic is not in the same premises as the health clinic' (7.5\%) and 'complex referral system' (6.8\%).

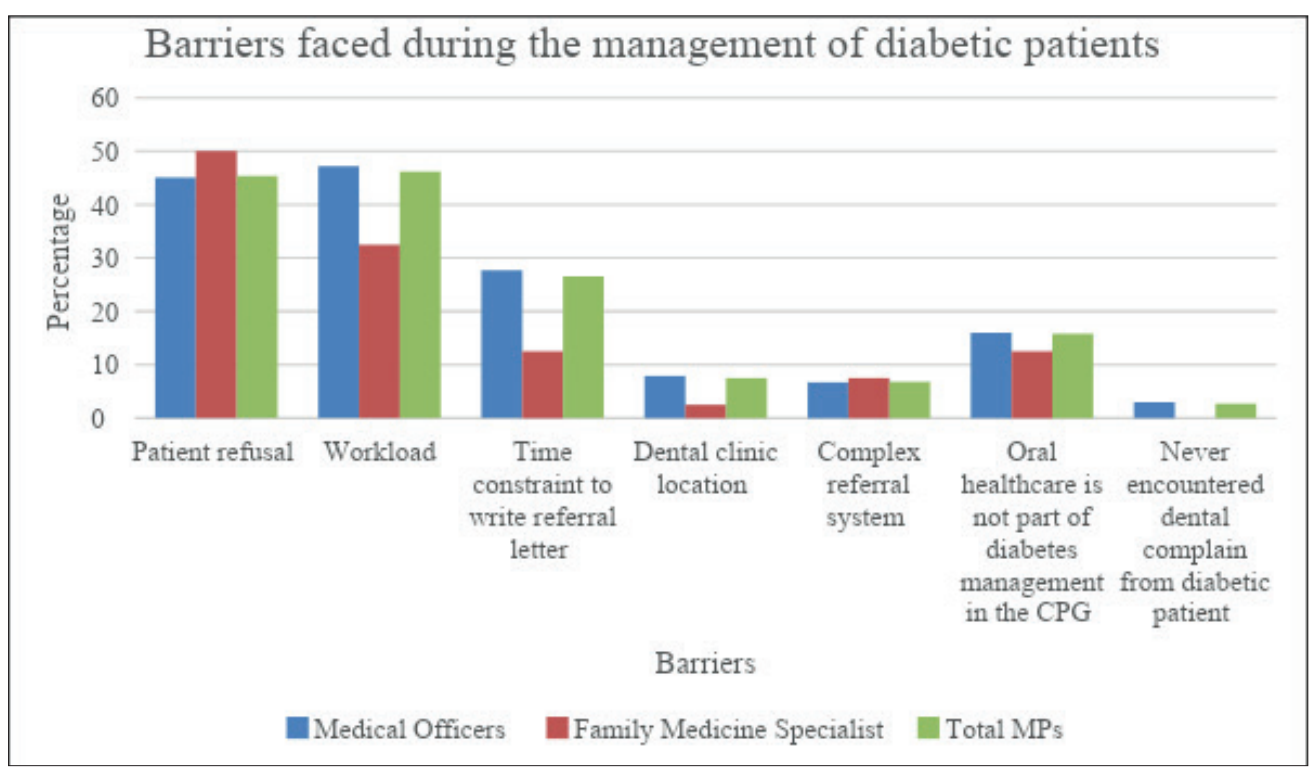

Figure 1. Barriers faced during the management of DM patients.

Apart from the barriers listed in Figure 1, the MPs listed additional perceived barriers. The most commonly noted barriers were that referral was only done when the patient requested it, there was no guideline or standard of practice (SOP) from dental to refer DM patients, and the MPs lacked knowledge and awareness. 
Table 6. Relationship between level of awareness, knowledge, attitude and practice scores and demographic characteristics.

\begin{tabular}{|c|c|c|c|c|}
\hline \multirow{2}{*}{$\begin{array}{l}\text { Domain } \\
\text { and variables }\end{array}$} & \multicolumn{2}{|c|}{ Simple logistic regression } & \multicolumn{2}{|c|}{ Multiple logistic regression } \\
\hline & $\begin{array}{l}\text { Crude OR } \\
(95 \% \mathrm{CI})\end{array}$ & $p$-value & $\begin{array}{l}\text { Adjusted OR } \\
(95 \% \mathrm{CI})\end{array}$ & $p$-value \\
\hline \multicolumn{5}{|l|}{ Awareness } \\
\hline Age(years old) & $4.8(0)$ & $<0.001$ & $0.91(0.82,1.01)$ & 0.087 \\
\hline $\begin{array}{l}\text { Gender } \\
\text { Male* } \\
\text { Female }\end{array}$ & $\begin{array}{c}1 \\
0.52(0.32,0.84)\end{array}$ & 0.007 & $\begin{array}{c}1 \\
0.53(0.32,0.88)\end{array}$ & 0.014 \\
\hline $\begin{array}{l}\text { Ethnicity } \\
\text { Malay* } \\
\text { Chinese } \\
\text { Indian }\end{array}$ & $\begin{array}{c}1 \\
1.53(0.70,3.34) \\
1.09(0.13,9.50)\end{array}$ & $\begin{array}{c}0.702 \\
0.13 \\
\end{array}$ & $\begin{array}{c}1 \\
0.090(0.09,9.85) \\
0.977(0.86,1,11) \\
\end{array}$ & $\begin{array}{l}0.933 \\
0.974\end{array}$ \\
\hline $\begin{array}{l}\text { Profession } \\
\text { Medical Officers* } \\
\text { FM Specialist }\end{array}$ & $\begin{array}{c}1 \\
0.11(0.02,0.84)\end{array}$ & 0.33 & & \\
\hline Years of service & $4.8(0)$ & ${ }^{*}<0.001$ & $1.02(0.59,1.75)$ & 0.94 \\
\hline $\begin{array}{l}\text { Smoking status } \\
\text { Smoker* } \\
\text { Non-smoker }\end{array}$ & $\begin{array}{c}1 \\
0.48(0.12,1.89)\end{array}$ & 0.290 & & \\
\hline $\begin{array}{l}\text { Perceived OH status } \\
\text { Poor* } \\
\text { Very Good } \\
\text { Good } \\
\text { Fair }\end{array}$ & $\begin{array}{c}1 \\
5.00(0.83,30.07) \\
2.42(0.60,9.73) \\
1.59(0.395,6.40)\end{array}$ & $\begin{array}{l}0.081 \\
0.214 \\
0.512\end{array}$ & $\begin{array}{c}1 \\
4.74(0.742,30.27) \\
2.40(0.56,10.23) \\
1.57(0.37,6.72) \\
\end{array}$ & $\begin{array}{l}0.100 \\
0.237 \\
0.541\end{array}$ \\
\hline \multicolumn{5}{|l|}{ Knowledge } \\
\hline Age(years old) & $0.95(0.86,1.05)$ & 0.334 & & \\
\hline $\begin{array}{l}\text { Gender } \\
\text { Male* } \\
\text { Female }\end{array}$ & $\begin{array}{c}1 \\
2.68(0.48,14.94) \\
\end{array}$ & 0.263 & & \\
\hline $\begin{array}{l}\text { Ethnicity } \\
\text { Malay* } \\
\text { Chinese } \\
\text { Indian }\end{array}$ & $\begin{array}{c}1 \\
0.25(0.076,0.85) \\
0.35(0.08,1.05) \\
\end{array}$ & $\begin{array}{l}0.076 \\
0.052\end{array}$ & $\begin{array}{c}1 \\
0.79(0.61,1.02) \\
0.63(0.00,-) \\
\end{array}$ & $\begin{array}{l}\mathbf{0 . 0 1 9} \\
1.000 \\
\end{array}$ \\
\hline $\begin{array}{l}\text { Profession } \\
\text { Medical Officers* } \\
\text { FM Specialist }\end{array}$ & $\begin{array}{c}1 \\
0.00\end{array}$ & 0.999 & & \\
\hline Years of service & $1.07(0.94,1.22)$ & 0.311 & & \\
\hline $\begin{array}{l}\text { Smoking status } \\
\text { Smoker* } \\
\text { Non-smoker }\end{array}$ & $\begin{array}{c}1 \\
0.23(0.026,1.91)\end{array}$ & 0.171 & $\begin{array}{c}1 \\
4.93(0.53,45.77) \\
\end{array}$ & 0.160 \\
\hline $\begin{array}{l}\text { Perceived OH status } \\
\text { Poor* } \\
\text { Very Good } \\
\text { Good } \\
\text { Fair }\end{array}$ & $\begin{array}{c}1 \\
10.68(2.73,41.75) \\
6.27(1.72,22.85) \\
2.42(0.60,9.73)\end{array}$ & $\begin{array}{l}0.45 \\
0.33 \\
0.23\end{array}$ & & \\
\hline \multicolumn{5}{|l|}{ Attitude } \\
\hline Age(years old) & $1.00(0.94,1.08)$ & 0.98 & & \\
\hline $\begin{array}{l}\text { Gender } \\
\text { Male* } \\
\text { Female } \\
\end{array}$ & $\begin{array}{c}1 \\
1.05(0.68,1.62)\end{array}$ & 0.83 & & \\
\hline $\begin{array}{l}\text { Ethnicity } \\
\text { Malay* } \\
\text { Chinese } \\
\text { Indian } \\
\end{array}$ & $\begin{array}{c}1 \\
0.64(0.28,1.45) \\
1.24(0.71,2.18) \\
\end{array}$ & $\begin{array}{l}0.46 \\
0.28 \\
\end{array}$ & & \\
\hline $\begin{array}{l}\text { Profession } \\
\text { Medical Officers* } \\
\text { FM Specialist }\end{array}$ & $\begin{array}{c}1 \\
1.17(0.48,2.83) \\
\end{array}$ & 0.73 & & \\
\hline Years of service & $1.00(0.69,1.47)$ & 0.98 & & \\
\hline $\begin{array}{l}\text { Smoking status } \\
\text { Smoker* } \\
\text { Non-smoker }\end{array}$ & $\begin{array}{c}1 \\
0.92(0.23,3.74) \\
\end{array}$ & 0.90 & & \\
\hline $\begin{array}{l}\text { Perceived OH status } \\
\text { Poor* } \\
\text { Very Good } \\
\text { Good } \\
\text { Fair }\end{array}$ & $\begin{array}{c}1 \\
0.85(0.18,3.98) \\
1.12(0.28,4.47) \\
0.95(0.24,3.83) \\
\end{array}$ & $\begin{array}{l}0.84 \\
0.87 \\
0.94 \\
\end{array}$ & & \\
\hline
\end{tabular}




\begin{tabular}{|c|c|c|c|c|}
\hline \multirow{2}{*}{$\begin{array}{l}\text { Domain } \\
\text { and variables }\end{array}$} & \multicolumn{2}{|c|}{ Simple logistic regression } & \multicolumn{2}{|c|}{ Multiple logistic regression } \\
\hline & $\begin{array}{l}\text { Crude OR } \\
(95 \% \mathrm{CI}) \\
\end{array}$ & $p$-value & $\begin{array}{c}\text { Adjusted OR } \\
(95 \% \mathrm{CI})\end{array}$ & $p$-value \\
\hline \multicolumn{5}{|l|}{ Practice referral } \\
\hline Age(years old) & $1.00(0.93,1.08)$ & 0.99 & & \\
\hline $\begin{array}{l}\text { Gender } \\
\text { Male* } \\
\text { Female }\end{array}$ & $1.69(1.07,2.68)$ & 0.25 & $\begin{array}{c}1 \\
1.70(1.08,2.68) \\
\end{array}$ & 0.073 \\
\hline $\begin{array}{l}\text { Ethnicity } \\
\text { Malay* } \\
\text { Chinese } \\
\text { Indian }\end{array}$ & $\begin{array}{c}1 \\
1.87(0.88,3.95) \\
1.08(0.57,2.04) \\
\end{array}$ & $\begin{array}{c}0.1 \\
0.81 \\
\end{array}$ & $\begin{array}{c}1 \\
0.94(0.16,5.48) \\
1.11(0.17,7.26)\end{array}$ & $\begin{array}{l}0.948 \\
0.917 \\
\end{array}$ \\
\hline $\begin{array}{l}\text { Profession } \\
\text { Medical Officers* } \\
\text { FM Specialist }\end{array}$ & $\begin{array}{c}1 \\
3.11(1.22,7.94) \\
\end{array}$ & 0.018 & $\begin{array}{c}1 \\
5.73(2.73,12.01)\end{array}$ & $<0.001$ \\
\hline Years of service & $1.07(0.71,1.61)$ & 0.75 & & \\
\hline $\begin{array}{l}\text { Smoking status } \\
\text { Smoker* } \\
\text { Non-smoker }\end{array}$ & $\begin{array}{c}1 \\
2.56(0.68,9.56)\end{array}$ & 0.16 & $\begin{array}{c}1 \\
0.24(0.06,0.98)\end{array}$ & 0.047 \\
\hline $\begin{array}{l}\text { Perceived OH status } \\
\text { Poor* } \\
\text { Very Good } \\
\text { Good } \\
\text { Fair }\end{array}$ & $\begin{array}{c}1 \\
10.15(1.13,91.44) \\
3.38(0.41,27.85) \\
2.48(0.30,20.65) \\
\end{array}$ & $\begin{array}{c}0.039 \\
0.26 \\
0.40 \\
\end{array}$ & $\begin{array}{c}1 \\
10.39(1.16,93.43) \\
3.45(0.42,28.38) \\
2.53(0.30,21.01) \\
\end{array}$ & $\begin{array}{l}\mathbf{0 . 0 3 7} \\
0.250 \\
0.391 \\
\end{array}$ \\
\hline
\end{tabular}

Note: $\mathrm{FM}=$ Family Medicine; $\mathrm{CI}=$ Confidence interval; $\mathrm{OR}=$ Odds Ratio; $\mathrm{OH}=$ Oral hygiene; $*$ indicates reference group.

Table 6 presents the relationship between the level of awareness, KAP score and demographic characteristics. Simple logistic regression provided preliminary results on potential associated factors ( $\mathrm{p}$-value $<0.25) .{ }^{19}$ Regarding awareness level, five variables (age, gender, ethnicity, years of service and perceived oral health $(\mathrm{OH})$ status) were found to be potentially significant. However, only gender and perceived $\mathrm{OH}$ status was found to be significant following multiple regression analysis. The odds of having high awareness were higher in female respondents (adjusted odds ratio $(\mathrm{aOR})=0.53,95 \% \mathrm{CI}=$ $0.32-0.86$ ) (Table 6) and in respondents with perceived good $\mathrm{OH}$ status $(\mathrm{aOR}=1.58,95 \%$ $\mathrm{CI}=1.09-2.28)$.

Regarding knowledge level, three variables (ethnicity, smoking status and perceived $\mathrm{OH}$ status) were found to be potentially significant. Based on the multiple regression analysis, only ethnicity was found to be significant $(\mathrm{aOR}=$ $0.79,95 \% \mathrm{CI}=0.61-1.02)$. No factors were found to be significant for attitude level. When considering the relationship between referral patterns and respondent characteristics in the present study, a significant relationship was observed between the MPs ethnicity $(\mathrm{aOR}=$ $0.79,95 \% \mathrm{CI}=0.61-1.02)$ and perceived oral health status $(\mathrm{aOR}=1.71,95 \% \mathrm{CI}=1.25$ 2.36) with patient referral.

\section{Discussion}

Our findings demonstrated good awareness status (80.6\%) among MPs on the bidirectional relationship of DM and PD. Studies conducted in New York $^{10}$ and Hong Kong $^{7}$ involving either trainee specialists or FMSs reported higher levels of awareness (97 and 92.1\%, respectively) that were not associated with age, years of experience, training status, demography or oral health behaviour. 7 The findings from both studies are comparable to those of the FMS group in the present study, where $92.5 \%$ were aware of this relationship. The favourable results for awareness among FMSs may be due to the increased knowledge and clinical experience they possess in managing DM patients.

In the present study, the majority of MPs were able to provide satisfactory responses to questions related to PD knowledge. This may be because these MPs were updated in their knowledge regarding $\mathrm{PD}$, which may have also been covered during their medical training or professional short courses conducted by the MOH, Malaysia. Most MPs had good knowledge of the aetiology, symptoms and risk factors of PD. Similar findings were also reported by a study in Turkey, where $87 \%$ of the medical doctors interviewed had knowledge about the symptoms of PD, whilst $59 \%$ of them knew that the primary clinical symptom of PD was bleeding of the gums. ${ }^{20}$

In our study, only $3.1 \%$ knew that excessive sugar intake was not an aetiology for PD. This finding was comparable to a study comprising 
obstetricians in North Carolina, who provided answers such as bacteria (94\%) and excess dietary sugar $(80 \%)$ as an aetiology of PD. ${ }^{21}$ These findings suggest that MPs may have a vague understanding of the pathogenesis of PD, which may be attributed to limited exposure to oral health during medical training. Ahmad et al. reported that medical schools in Australia and Malaysia did not offer specific learning modules on oral health but instead offered oral health education as an integrated component of the medical curriculum in the preclinical and clinical years. ${ }^{22}$

Regarding the attitudes of MPs, only $31.4 \%$ exhibited positive attitudes towards PD in the management of DM. The higher negative attitude scores in this study were shown to disagree with the good knowledge scores on PD. This is in contrast to the findings of Quijano et al., which noted a positive correlation between self-rated knowledge and total attitude score. ${ }^{10}$ Overall, $89.6 \%$ of the MPs had a positive attitude when asked about the importance of their management of DM patients with PD. These positive attitudes were mostly attributed to the good knowledge and awareness of the relationship between both diseases. These findings were compared to the findings from a study in North Carolina, in which $88 \%$ of respondents agreed that physicians should be taught about PD and trained to screen patients with PD.

Regarding the practices of the MPs, most were found to have 'poor practice' scores. However, when comparing between the groups, the FMSs exhibited significantly higher 'good practice' scores than the MOs. Although $97.4 \%$ of the MPs had good knowledge scores, only $30.2 \%$ asked their patients about symptoms of $\mathrm{PD}$ and only $34.2 \%$ referred their DM patients to the dentist. FMSs enquired (50\%) and referred (67.5\%) more than MOs (28.7 and 31\%, respectively). However, the MPs in this study had a higher level of enquiry for their patients when compared to the findings of Quijano et al., which noted that only $18 \%$ of American internal medical trainees asked their patients about whether they were diagnosed with PD and only $24 \%$ screened their patients for the disease. ${ }^{10}$ Similar to our findings, another study of health care workers (HCWs) in Malaysia also reported a low referral pattern for DM patients to dentists (26\%). ${ }^{15}$ This was despite the fact that the majority of their respondents agreed that the referral of DM patients for routine dental check-ups was necessary. In the present study, one reason for the poor referral rate to dentists could be the poor attitudes among MPs regarding PD management for their DM patients.

Despite MPs' good awareness and knowledge status in the present study, lower patient recommendation and referral patterns were observed. This phenomenon was also observed in a few other studies from different parts of the world. ${ }^{7,8,10,20}$ To improve and promote oral health care in the population, it is vital to investigate, identify and address the key barriers that prevent access to dental care. For example, an Iranian study on hand hygiene found poor hand hygiene practices among the studied residents despite having acceptable knowledge of proper hand hygiene. The main reasons given by the residents were time constraints and heavy workload. ${ }^{23}$ In the current study, MPs' knowledge and awareness status was observed to be good but did not translate into practice.

Our findings suggest that Malays and MPs who perceived good $\mathrm{OH}$ were more likely to refer when compared to other ethnic groups and respondents who perceived their oral hygiene as poor. This finding is in line with a study from Hong Kong that reported patient referral being mostly associated with MPs' $\mathrm{OH}$ practices and perceived $\mathrm{OH}$ status. ${ }^{7}$ In Malaysia, the heavy workload in primary care clinics is mostly attributed to the low doctor-to-patient ratio, which may also contribute to poor patient referrals. Although the number of doctors in Malaysia increased by $7.7 \%$ in 2016, the doctorto-population ratio remained high (1 doctor: 632 persons $)^{24}$ despite the government target of 1 doctor: 400 persons in 2019. ${ }^{25}$

MPs also listed a few perceived barriers that they experienced during the management of DM patients. The most common barriers included referrals only being performed when patients requested them, no guidelines or SOPs from the dental fraternity for referral, and MPs lacking knowledge and awareness. Similar findings were reported in a study by Salleh et al., which found that $40 \%$ of the studied HCWs performed referrals when requested by their patients, whilst $44.4 \%$ of the HCWs requested a simple referral system.

\section{Limitations}

This study has certain limitations. First, the 
convenience sampling method used in this study leads to generalisability issues. Notably, the findings cannot be generalised to the population of interest since this research was only conducted in a $\mathrm{MOH}$ public primary care setting in selected states within Peninsular Malaysia. There is also the possibility of under-representing the studied population and the potential for voluntary response bias. As of October 2017, a total of 104 FMSs in Malaysia were serving in primary care clinics in selected states. However, only 40 FMSs were involved in this study. Secondly, the number of MOs who participated in this study was higher than the number of FMSs. This is expected since there are only one or two FMSs posted in each primary care clinic and a higher number of MOs present in clinics. We are aware that a small sample size may affect the precision of the measurement. Nevertheless, this finding provides insights into the issue at hand. Third, the validity of the questionnaire used in this study was only tested using content and face validity. Since this study is considered a pilot study, the validity testing is deemed sufficient. However, it is recommended that the questionnaire be tested for construct validity for future use. Another limitation of this study is that the reliability of the questionnaire was not tested before data collection due to time constraints and anticipated difficulty in obtaining a sufficient sample size. This raised the question of whether or not the findings are reliable. To obtain some insights into the reliability of this study, internal consistency testing was performed on the data. It was found that the Cronbach's alpha values were 0.24 (attitude), 0.40 (knowledge), 0.48 (awareness) and 0.73 (practice). Besides the practice domain, Cronbach's alpha values for all other domains were below 0.7. Thus, the reliability of the questionnaire is questionable. Therefore, the findings of this study need to be interpreted with caution.
Since PD is a silent disease, not many patients realise that they are suffering from it. Our findings indicate that MPs have adequate knowledge about PD and its relationship with DM. Notably, this knowledge should be shared with DM patients through dental education provided during routine health education programmes. To reinforce this, future CPGs on DM management should include adequate information on the bi-directional relationship of both diseases and their complications. A proper SOP on DM patient referrals should also be emphasised in the CPG. Moreover, a simple referral format can be developed by dental policymakers. The incorporation of a specific module on the relationship between oral health and systemic diseases in the undergraduate medical curriculum may be beneficial and improve MPs' KAP related to this relationship.

\section{Conclusion}

In this study, most MPs were aware of the association between DM and PD and had good knowledge of PD. Despite having good knowledge and awareness, most MPs had poor attitudes in their management of DM patients with PD. Additionally, patients may not benefit from the MPs' awareness and knowledge since it was seldom put into practice. Thus, further measures to improve MPs' attitudes and practices whilst enhancing patients' knowledge on DM and PD should be explored. Moreover, identified barriers should be addressed accordingly.

\section{Acknowledgements}

This study was supported by the Dental Postgraduate Research Grant (DRPG/11/17) from the University of Malaya. We would like to thank the Ministry of Health of Malaysia for allowing us to conduct this study. The authors have no conflicts of interest to declare.

\section{How does this paper make a difference to general practice?}

- The importance of using a multidisciplinary approach to managing diabetes mellitus (DM) patients with periodontitis (PD) amongst Malaysian medical practitioners (MPs) (family medicine specialists and medical officers) is emphasised.

- Although MPs had good knowledge of PD, there was room for improvement in their attitudes and practices in managing these patients.

- An increase in MPs' awareness of PD will lead to early PD detection and thus more favourable treatment responses for both DM and PD.

- Information on the management of DM patients with PD should be incorporated into the Malaysian Clinical Practice Guidelines (CPG) on the management of DM patients. 


\section{References}

1. Kassebaum NJ, Bernabé E, Dahiya M, et al. Global burden of severe periodontitis in 1990-2010: A systematic review and metaregression. J Dent Res. 2014;93(11):1045-53.

2. Nelson RG, Shlossman M, Budding LM, et al. Periodontal disease and NIDDM in Pima Indians. Diabet Care. 1990; 13:836-40.

3. Graziani F, Gennai S, Solini A, et al. A systematic review and meta-analysis of epidemiologic observational evidence on the effect of periodontitis on diabetes: An update of the EFP-AAP review. J Clinical Periodontol. 2018;45(2):167-87.

4. Madianos PN, Koromantzos PA. An update of the evidence on the potential impact of periodontal therapy on diabetes outcomes. J Clin Periodontol. 2018;45(2):188-95.

5. Sanz M, Ceriello A, Buysschaert M, et al. Scientific evidence on the links between periodontal diseases and diabetes: Consensus report and guidelines of the joint workshop on periodontal diseases and diabetes by the International Diabetes Federation and the European Federation of Periodontology. Diabetes Res Clin Pract. 2018; 137:231-41.

6. Management of Type 2 Diabetes Mellitus [Internet]. Ministry of Health, Malaysia 2015. Available from: http://www.moh.gov.my/ penerbitan/CPG/CPG\%20T2DM\%202015. pdf.

7. Tse SY. Diabetes mellitus and periodontal disease: Awareness and practice among doctors working in public general out-patient clinics in Kowloon West Cluster of Hong Kong. BMC Fam Pract. 2018;19(1):199.

8. Al-Habashneh R, Barghout N, Humbert $\mathrm{L}$, et al. Diabetes and oral health: Doctors' knowledge, perception and practices. J Eval Clin Pract. 2010;16(5):976-80.
9. Owens JB, Wilder RS, Southerland JH, et al. North Carolina internists' and endocrinologists' knowledge, opinions, and behaviors regarding periodontal disease and diabetes: Need and opportunity for interprofessional education. J Dent Educ. 2011;75(3):329-38.

10. Quijano A, Shah AJ, Schwarcz AI, et al. Knowledge and orientations of internal medicine trainees toward periodontal disease. J Periodontol. 2010;81(3):359-63.

11. Bissett SM, Stone KM, Rapley T, et al. An exploratory qualitative interview study about collaboration between medicine and dentistry in relation to diabetes management. BMJ Open. 2013;3(2):e002192.

12. Lin H, Zhang H, Yan Y, et al. Knowledge, awareness, and behaviors of endocrinologists and dentists for the relationship between diabetes and periodontitis. Diabetes Res Clin Pract. 2014;106(3):428-34.

13. Chitta H, Chaintanya N, Lavanya R, et al. Awareness of medical doctors on oral health: A cross sectional study. Oral Health Dent Manag. 2015;14(6):424-28.

14. Nam S, Chesla C, Stotts NA, et al. Barriers to diabetes management: Patient and provider factors. Diabetes Res Clin Pract. 2011;93(1):1-9.

15. Salleh NC, Yaw SL, Muttalib KA, et al. Perception and trends in referral of diabetes patients for dental care among healthcare workers (HCWs). Int J Healthcare Sci. 2017;5(1):424-9.

16. Institute for Public Health. National Health \& Morbidity Survey. Vol II: Non-communicable Diseases, Risk Factors \& Other Health Problems. Malaysia, Ministry of Health, Malaysia; 2015.

17. Naing L, Winn T, Rusli B. Practical issues in calculating the sample size for prevalence studies. Arch Orofacial Sci. 2006;1(1):9-14.
18. Shwetha M, Prasad S, Anvitha D, et al. Awareness of the relationship between the periodontal health and systemic diseases among medical practitioners: A randomized questionnaire study. Indian J Appl Res. 2016;6(4):31-34.

19. Bursac Z, Gauss CH, Williams DK, et al. Purposeful selection of variables in logistic regression. Source Code Biol Med. 2008;3(1):17

20. Tasdemir Z, Alkan BA. Knowledge of medical doctors in Turkey about the relationship between periodontal disease and systemic health. Braz Oral Res. 2015;29(1):1-8.

21. Wilder R, Robinson C, Jared HL, et al. Obstetricians' knowledge and practice behaviors concerning periodontal health and preterm delivery and low birth weight. J Am Dent Hyg Assoc. 2007;81(4):81-.

22. Ahmad MS, Abuzar M, Razak I, et al. Educating medical students in oral health care: Current curriculum and future needs of institutions in Malaysia and Australia. Eur J Dent Educ. 2017;21(4):e29-e38.

23. Nabavi M, Alavi-Moghaddam M, Gachkar $\mathrm{L}$, et al. Knowledge, attitudes, and practices study on hand hygiene among Imam Hossein Hospital's residents in 2013. Iran Red Crescent Med J. 2015;17(10):1-8.

24. Social Statistics Bulletin, Malaysia, 2017 [Press release]. Malaysia; 2017.

25. Kheng KS. How many doctors does Malaysia really need? The Star. 2019. 